\title{
Research Paper \\ The Relationship between Sleep Quality and Depression in Older People Living in 3 Dis- tricts of Tehran, Iran
}

\section{*Ahmad Beh-Pajooh ${ }^{1}$, Simin Soleymani ${ }^{2}$}

1. Department of Psychology of Exceptional Children Education, Faculty of Psychology \& Education Sciences, University of Tehran, Tehran, Iran.

2. Department of Clinical Psychology, Faculty of Psychology \& Education Sciences, Science and Research Branch, Islamic Azad University, Zanjan, Iran.

Citation: Beh-Pajooh A, Soleymani S. [The relationship between sleep quality and depression in older people living in 3 districts of Tehran, Iran (Persian)]. Iranian Journal of Ageing. 2016; 11(4):72-79. http://dx.doi.org/10.21859/sija-110172

doi): http://dx.doi.org/10.21859/sija-110172

Received: 06 Dec. 2015 Accepted: 18 Feb. 2016

Key words: Sleep quality, Depression, People residing in nursery homes

\begin{abstract}
A B S T R A C T
Objectives Most old age people are facing physical and mental problems such as movement problems, chronic pain, sleeping problems, seclusion, and depression. Thus, it is necessary to allocate a good share of health and treatment resources in any society to old age people and attend their problems. This study aimed to examine the relationship between sleep quality and the level of depression in people residing in nursing homes of 3 districts of Tehran City, Iran, in 2013.

Methods \& Materials This descriptive-analytical study included people living in nursing homes of Tehran Welfare Organization. The study sample consisted of 90 older people who were selected through cluster random sampling considering the proportion of the northern, central, and southern regions of Tehran and observing their level of consciousness, ability to respond, and willingness to participate in the study. To obtain the data, we used 2 questionnaires of Pittsburgh sleep quality index and geriatric depression scale. Then, the obtained data were analyzed through multivariate regression.

Results Older women constituted $60 \%$ of the participants. The findings revealed that there was a negative and significant relationship $(\mathrm{P}<0.001)$ between sleep quality of the elderly people and their depression level. However, there was a positive and significant relationship $(P<0.001)$ between the level of depression and variables of sleep disorders, using sleep aids, and disorders in daily activities.

Conclusion There is a causal and mutual relationship between sleep disturbances and depression, in such a way that sleeplessness could end in depression, and if the depression signs are ignored, it will gradually affect sleep quality. Generally, sleep disturbances are mutually associated with depressive mood and low quality of life. Therefore, to enhance sleep quality and decrease the level of depression in people residing in nursing homes, the necessity of special care programs in the old age should be emphasized. With regard to limitations of this study, we cannot generalize our results to all old population as our study was confined to only 3 districts of Tehran. In addition, since some variables like education and economic status of old age people were not considered in this study, there is a need to generalize the findings. Finally, it is recommended that future studies should investigate the relationship between sleep quality, lifestyle, and daily activities in the nursing homes.
\end{abstract}

* Corresponding Author:

Ahmad Beh-Pajooh, PhD

Address: Department of Psychology of Exceptional Children Education, Faculty of Psychology \& Education Sciences, University of Tehran, Tehran, Iran. Tel: +98 (912) 3844236

E-mail: behpajooh@ut.ac.ir 


\title{
رابطه كيفيت خواب با افسردگى در افراد سالمند در سه ناحيه شهر تهران
}

\author{
"احمد بهيثوه'، سيمين سليمانى \\ 1- كروه روانشناسى و آموزش كودكان استثنايى، داتشكده روانشناسى و علوم تربيتى، دانشكاه تهران، تهران، ايران.

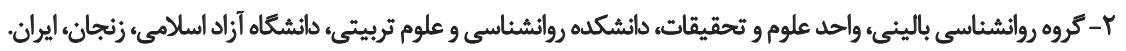

\begin{abstract}
حكSי

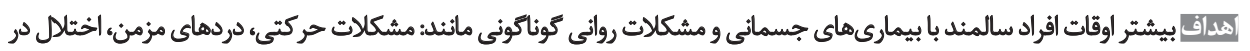

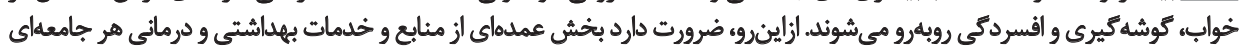

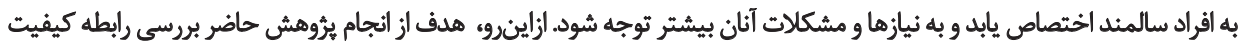

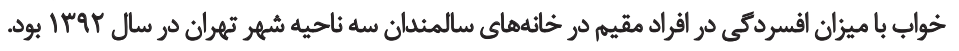

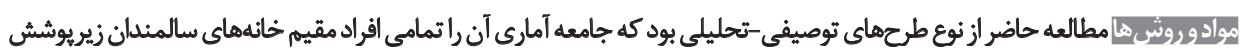

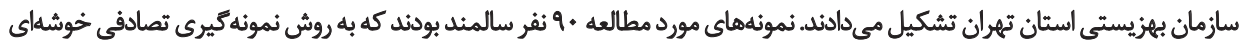

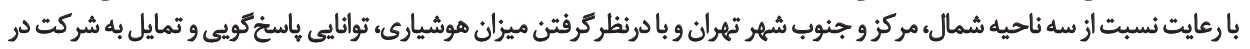

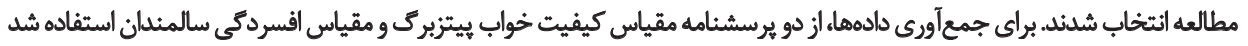

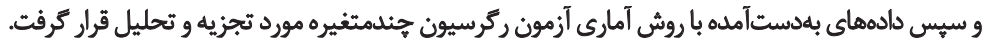

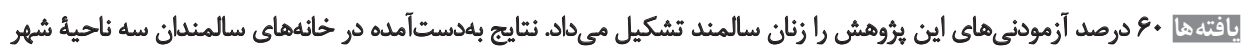

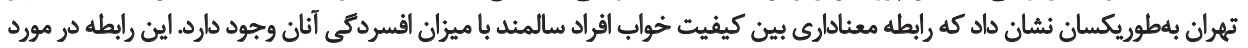

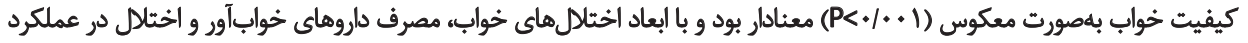

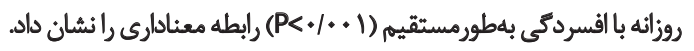

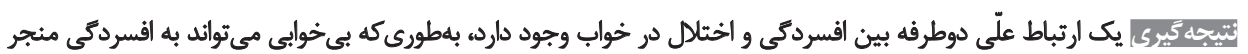

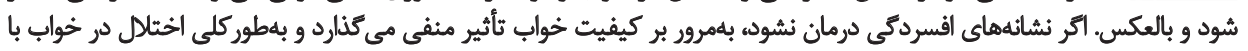

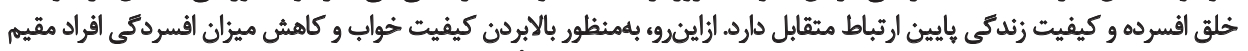

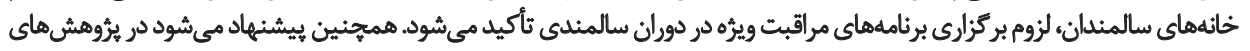

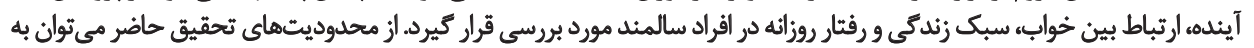

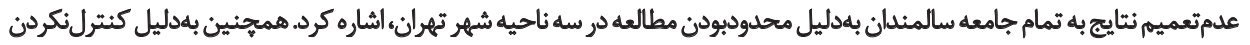

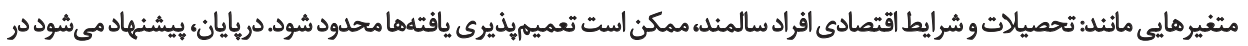

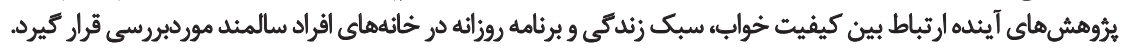

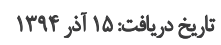

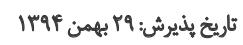

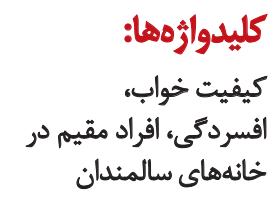

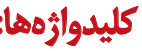

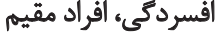

خانهماي سالمندان

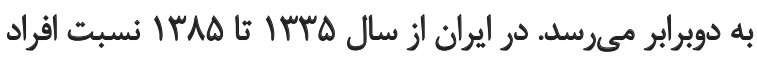

daleo

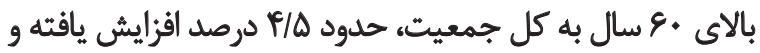

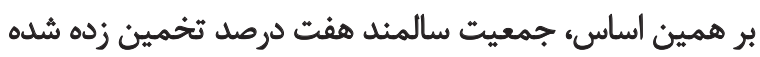

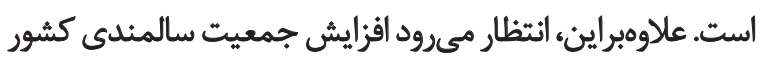

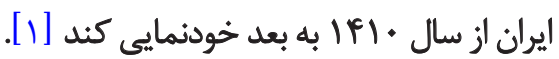

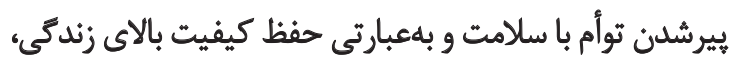

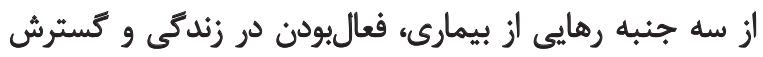

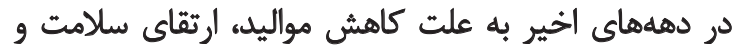

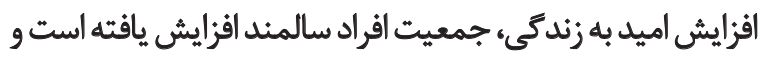

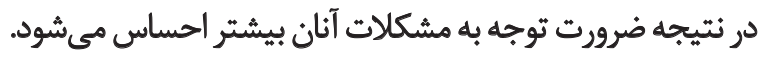

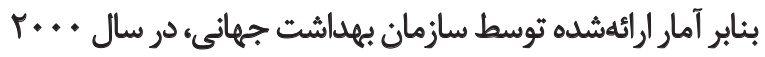

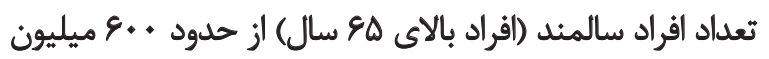

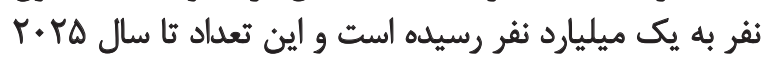

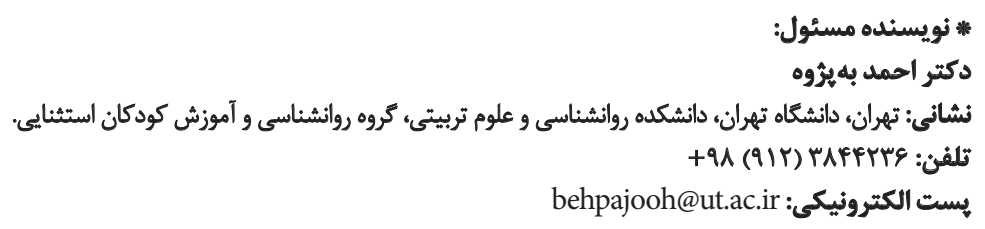




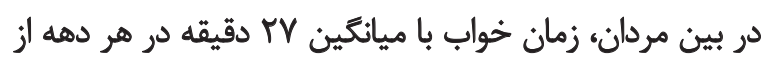

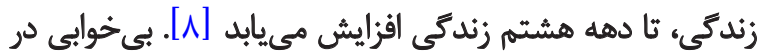

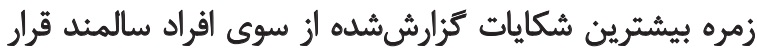

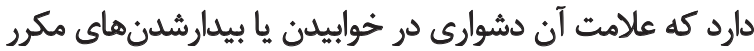

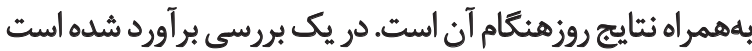

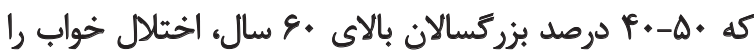

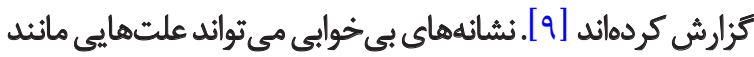

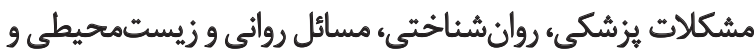

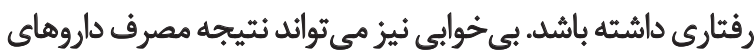

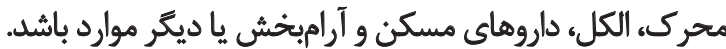
بيشتر افراد سالمند بهعلت دردهاى مزمن، دردهاى استخوانى

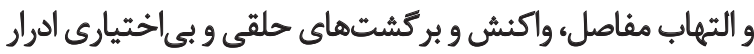

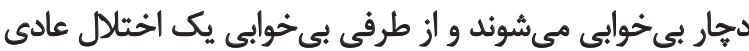

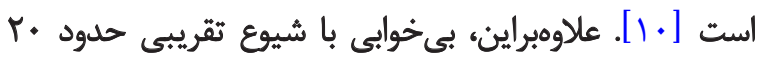

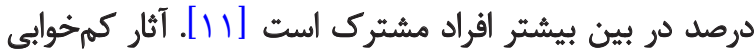

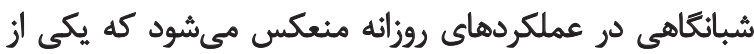

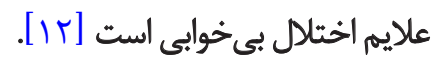

تضعيف عملكرد روزانه و بي خوابى شبانكاهي، بهعنوان علايم

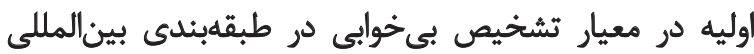

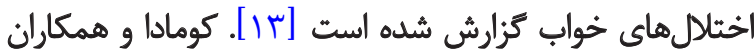
و (T.1T)

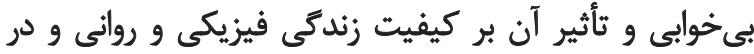

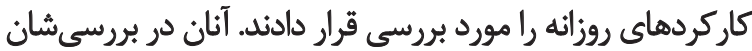

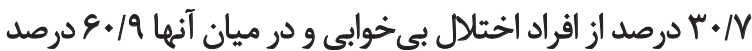

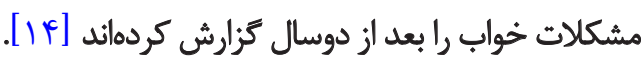

در برخى از تحقيقات كزارش شده كه بي خوابى مىتواند

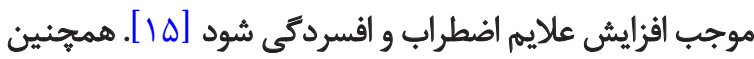

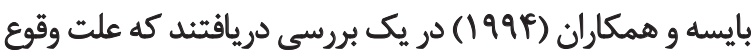

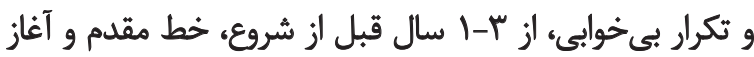

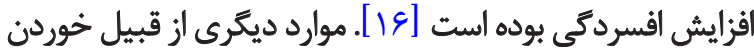

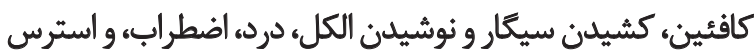

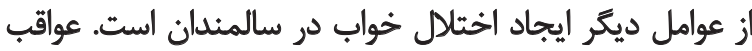

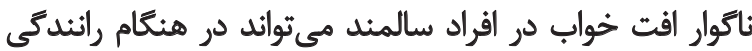

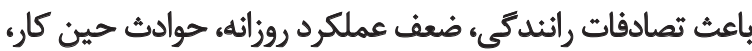

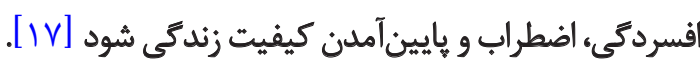
شايان ذكر است كه بررسى وضعيث سلامت افراد سالمند در در

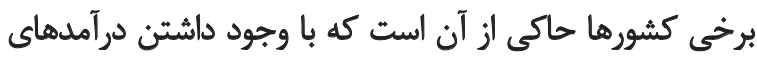

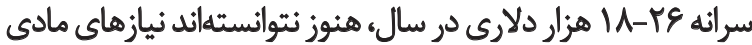

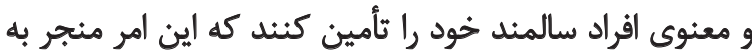

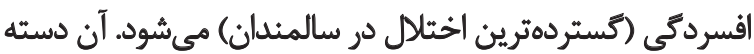
از علايمى كه بيشتر جنبه جسمانى دارنده مانند ضعف، خستختى

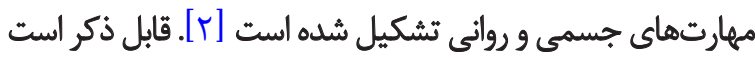

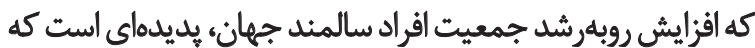

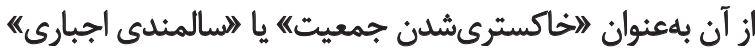

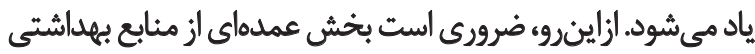

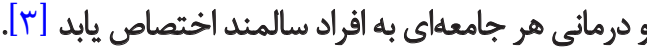

يديده سالمندى همواره با اختلالهاى جسمانى و رواثي

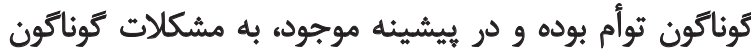

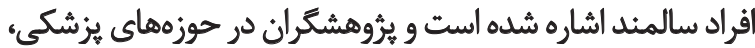

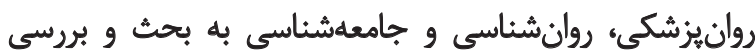

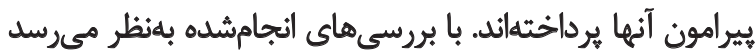

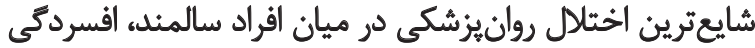

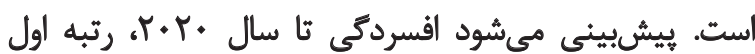

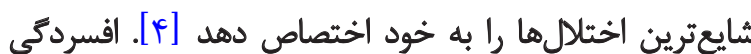

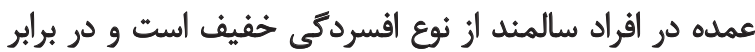

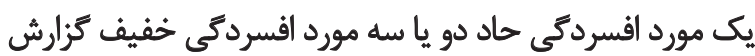

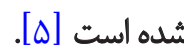

يكى ديكر از مشكلات رايج دوران سالمندى، اختلال خواب است.

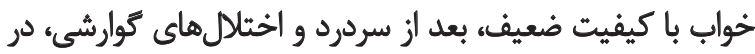

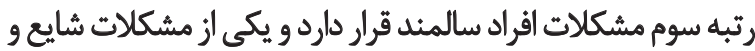

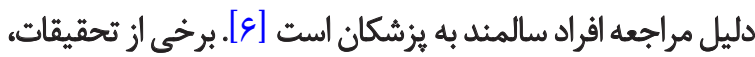

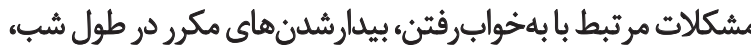

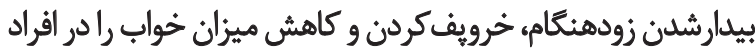

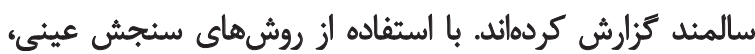

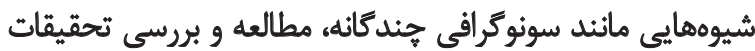

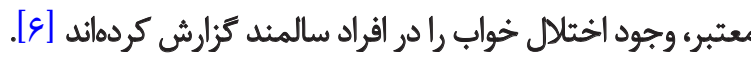

بررسى هاييى كه به مقايسه خواب اشخاص سالمند باديكر افراد

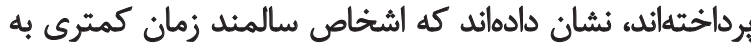

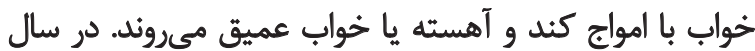

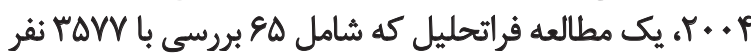

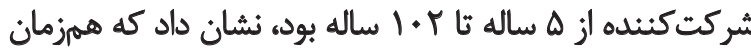

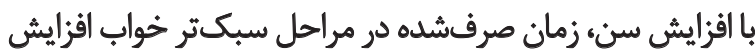

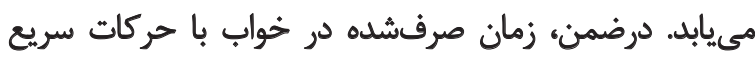

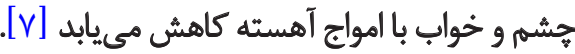

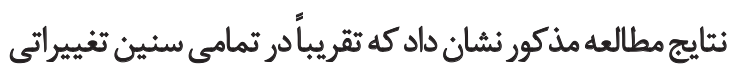

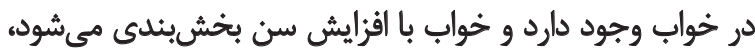

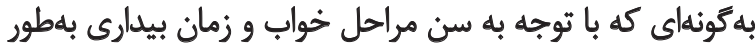

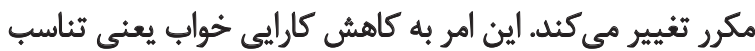

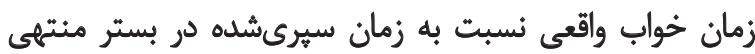

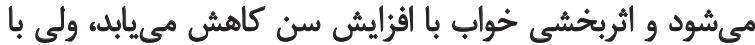

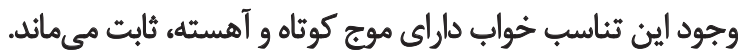

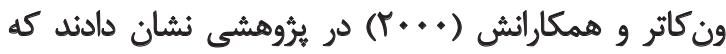




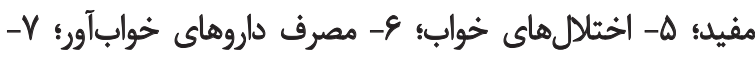

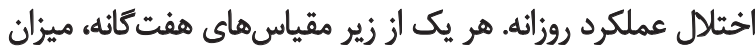

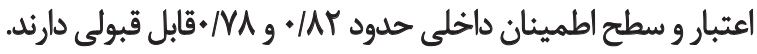

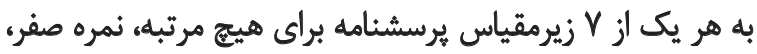

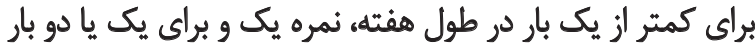

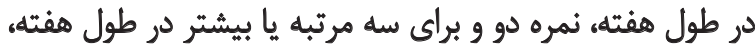

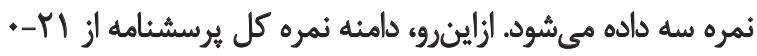

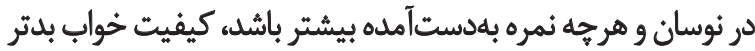

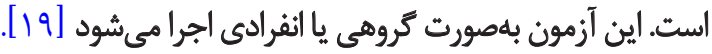

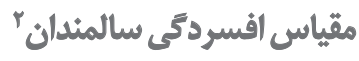

در ميان مقياسهاى موجود، مقياس افسردكى سالمندان

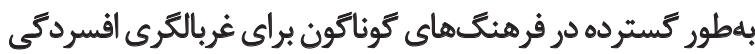

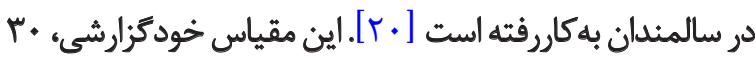
يرسش دارد كه آزمودنى با انتخاب تزينها

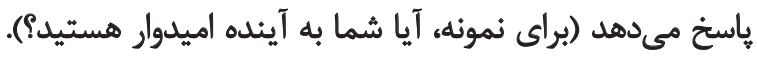

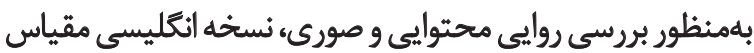

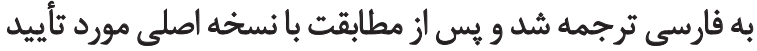
كارشناسان قرار ترفت.

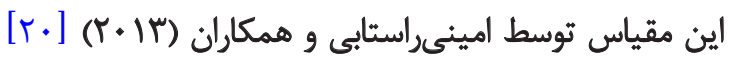

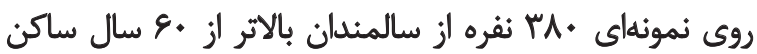

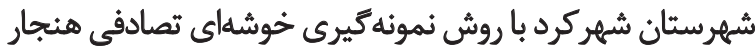

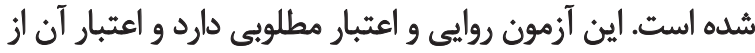

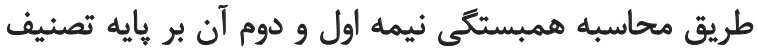

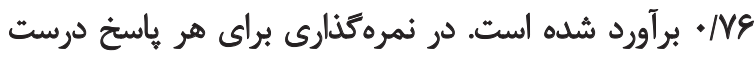

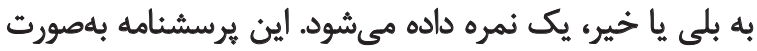

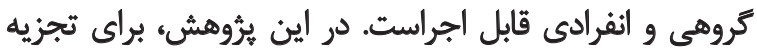

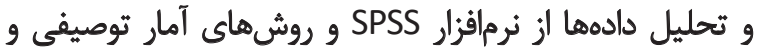
ركريون جندمتغيره استفاده شد.

يافتهها

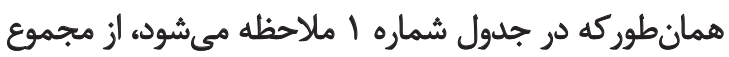

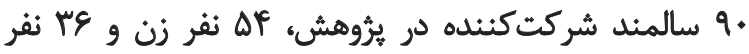

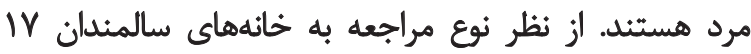

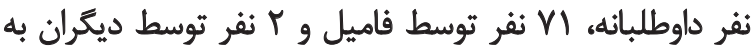

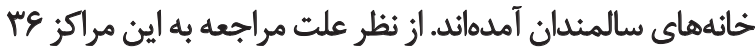

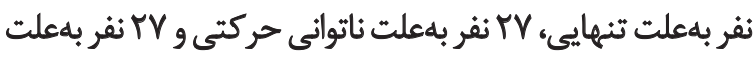

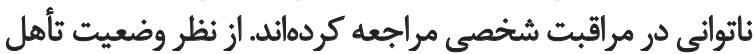

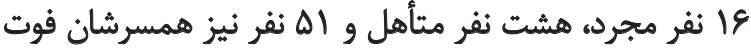

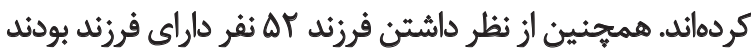

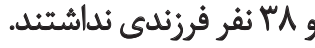

و بى اشتتهايى، وجود اشتغال ذهنى در ارتباط با سلامتى و توجه

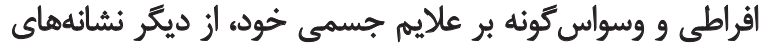

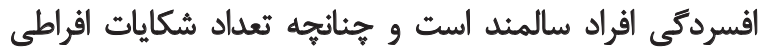

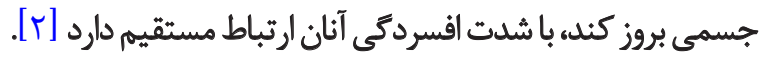
با توجه به بيشينه موجود مبنىبر شيوع اختلالهاى خواب

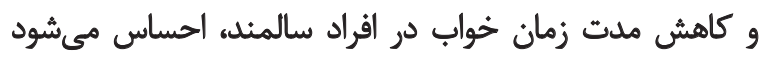

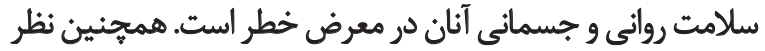

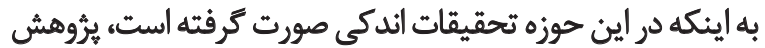

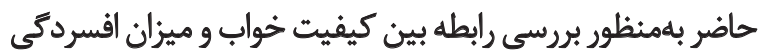

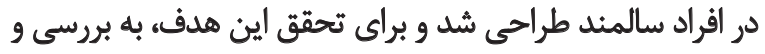

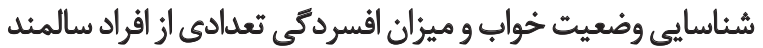
مقيم در خانههاي سالمندان سه ناحيه شهر تهران يرداخت. روش مطالعه

طرح يُروهش حاضر با توجه به ماهيت موضوع واهداف موردنظر،

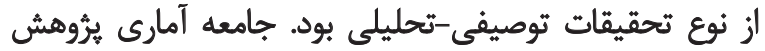

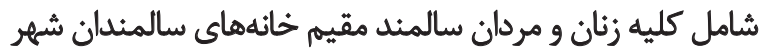

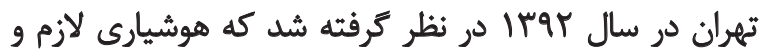

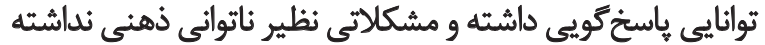

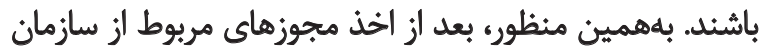

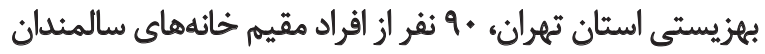

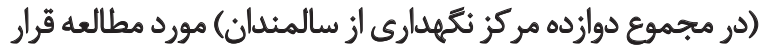

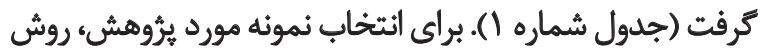

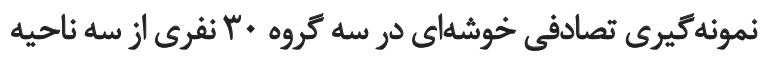

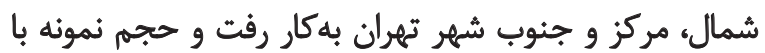

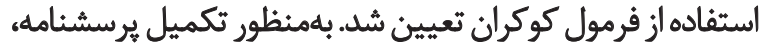

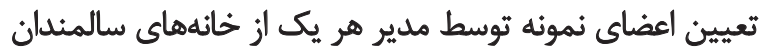

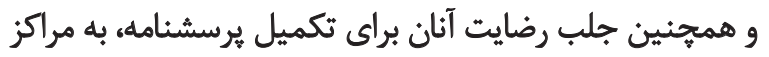

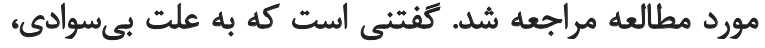

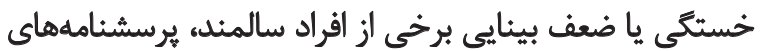

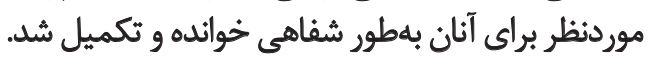

براى جمع آورى دادهها، از دو ابزار به شرح زير استفاده شد:

\section{مقياس كيفيت خُواب يبيزوبرى'}

اين يرسشنامه خودكزارشى با هدف بررسى كيفيت خواب توسط إئ

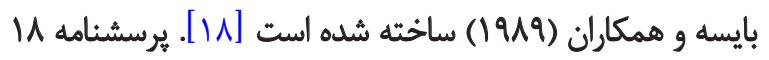

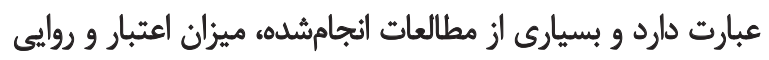

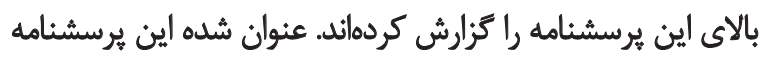

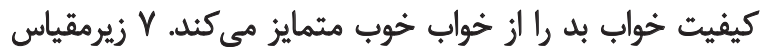

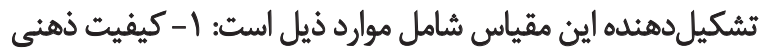

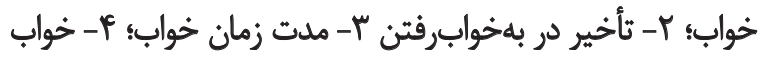

1. Pittsburgh Sleep Quality Index (ISQP) 
جدول ا. ويرّكى هاى جمعيتشناختى سالمندان شركت كننده در تحقيق به تفكيك جنسيت، نوع مراجعه، علت اقامت، وضعيت تأهل و تعداد فرزندان.

\begin{tabular}{|c|c|c|c|c|c|c|c|c|c|c|c|c|c|c|}
\hline \multirow{2}{*}{ هجموع } & \multicolumn{4}{|c|}{ جنوب - } & \multicolumn{4}{|c|}{ 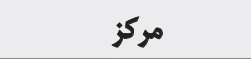 } & \multicolumn{4}{|c|}{ 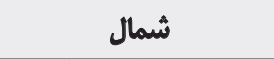 } & \multicolumn{2}{|c|}{ منطقه شُبرى } \\
\hline & ir & 11 & 1. & 9 & $\wedge$ & $v$ & $\varepsilon$ & $\Delta$ & r & r & $r$ & 1 & دان مورد مطالعه & خائهاي سالد \\
\hline q. & $\checkmark$ & If & 9 & $\checkmark$ & $\checkmark$ & $\Delta$ & 11 & 8 & $r$ & $v$ & $\wedge$ & $\&$ & يإسخدهنده & تعداد أفر \\
\hline$\Delta f$ & - & 1. & f & $\Delta$ & f & f & $\Delta$ & r & $r$ & 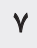 & 8 & f & ij.1 & \\
\hline re & $\checkmark$ & f & $\Delta$ & r & r & 1 & $\&$ & r & 1 & - & $r$ & r & Y. ير & יבצח \\
\hline IV & 1 & 1 & - & 1 & r & - & f & 1 & 1 & $r$ & $r$ & 1 & 1. خُودشان & \\
\hline$n$ & $\Delta$ & ir & 9 & 8 & $\Delta$ & $\Delta$ & $\checkmark$ & $\Delta$ & r & $p$ & 8 & $\Delta$ & ب. قاميل & توع مراجعه توبطط \\
\hline r & 1 & 1 & - & - & • & - & - & - & - & - & - & - & "َ. ديكران & \\
\hline re & 1 & $\Delta$ & $\Delta$ & - & $p$ & . & $\varphi$ & r & r & r & $\Delta$ & $\varphi$ & ا..تنهايي & \\
\hline rV & - & $\Delta$ & $r$ & $\Delta$ & - & r & - & r & - & $\Delta$ & $r$ & $r$ & ז. نائوائى حركتى & |قامت در خائه سالمندان \\
\hline rV & $\varepsilon$ & r & $r$ & $r$ & r & $r$ & $\checkmark$ & . & . & - & $\cdot$ & - & ". ناتواني در مراقبت شخصي & \\
\hline 18 & $\varphi$ & 1 & $\Delta$ & 1 & 1 & . & + & . & 1 & . & $r$ & 1 & |. مجرد & \\
\hline$\Lambda$ & . & 1 & 1 & . & . & 1 & $r$ & 1 & + & 1 & + & + & 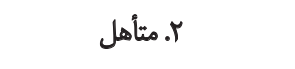 & ضضعدت تأهي \\
\hline ه) & r & ir & 1 & 8 & $r$ & f & $\Delta$ & $f$ & 1 & 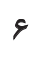 & r & f & بـ بوت همسر & وصنيك ناهل \\
\hline 10 & 1 & $\cdot$ & $r$ & . & $f$ & . & $r$ & 1 & 1 & . & $r$ & 1 & f. متاركه & \\
\hline$\Delta r$ & $r$ & 1. & $r$ & $r$ & r & $\Delta$ & 1. & $\Delta$ & $r$ & $f$ & $r$ & $r$ & ا ا. دارند & \\
\hline rA & $\Delta$ & f & 8 & f & $p$ & - & 1 & 1 & 1 & $r$ & $\&$ & $r$ & ז. ندارند & 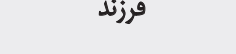 \\
\hline
\end{tabular}

كالم

همان طور كه در جدول شماره ب مشاهده مي شود بين افسردكي و

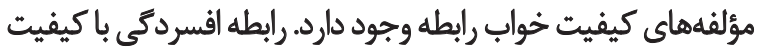

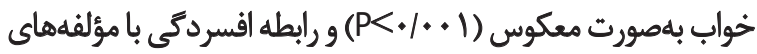

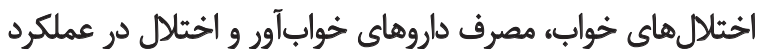

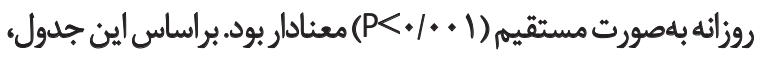
سالمندان مقيم سراى سالمندان علاوهبر افسردكى، با اختلال هاى

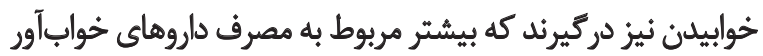

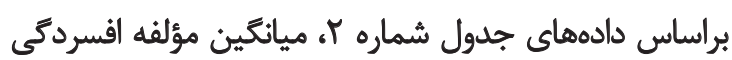

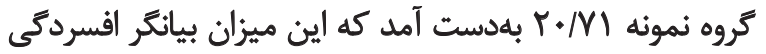

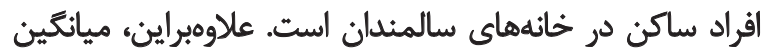

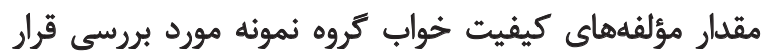

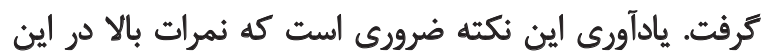

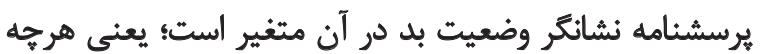

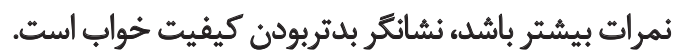

جدول r. آمار توصيفى مربوط به متغيرهاى ثُؤهش (تعداد م9 نفر).

\begin{tabular}{|c|c|c|}
\hline انجحراف معيار & مياتكين & متغير \\
\hline$\Delta / \cdot 1$ & $r \cdot M$ & أفسردكى \\
\hline. prr & $1 / K E$ & ا. كيفيت خواب \\
\hline .119 & V/P & Y. كيفيت ذهنى خواب \\
\hline .198 & $1 / T Y$ & م. تأخير در خواييلن \\
\hline$\cdot M$ & $r / T Q$ & fا. مدت خواب و خواب مفيد \\
\hline the &.$/ 99$ & هـ اختالالهاي خواب \\
\hline .19. & r/eV & f مصرف داروى خوابآور \\
\hline$\cdot|A|$ & V/T & V. اختلال در عملكرد روزائه \\
\hline
\end{tabular}

il 
جدول ץ. نتايج ركرسيون جيندكائه متغيره با هدف تعيين رابطه ميزان افسردكى و مؤلفهاي كيفيت خواب.

\begin{tabular}{|c|c|c|c|c|}
\hline سطح معنادارى & $\mathrm{t}$ & ضرايب استاندارد بتا & متغيرهاى مستقل & متغير وابسته \\
\hline$\%$ & q/IV & & اثر ثابت & \multirow{8}{*}{ افسردمى } \\
\hline$\%$ & $-\Delta / 9 \Delta f$ &.$-|9 T|$ & كيفيت خواب & \\
\hline . IDFA &.$- / 8 \cdot f$ & -.1 .94 & كيفيت ذهنى خواب & \\
\hline . MEA & $1 / 18$ & .1 .91 & تأخير در خوابيدن & \\
\hline $.119 \Delta$ & $1 / f+1$ & .1 .99 & طول مدت خواب و خواب مفيد & \\
\hline$\%$ & $\Delta / r u$ &.$/ P V A$ & اختلال هاى خواب & \\
\hline$\ldots \Delta$ & T/MV &.$/ 198$ & مصرف داروهاى خواب!آور & \\
\hline$\%$ & P/MY & . rar & اختالال هاى در عملكرد روزانه & \\
\hline
\end{tabular}

记

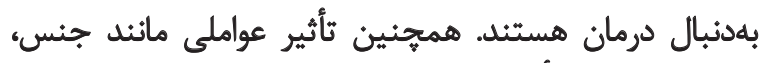

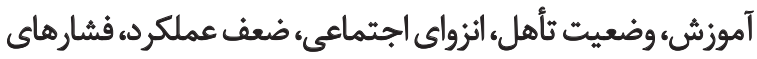

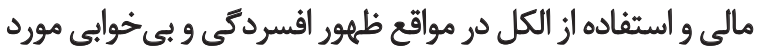

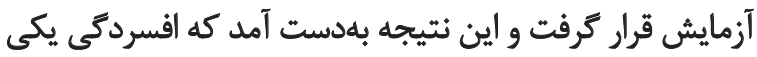

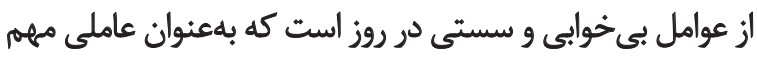

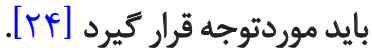

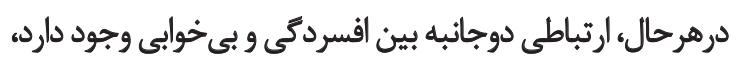

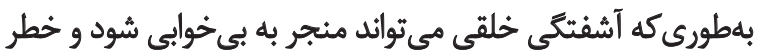

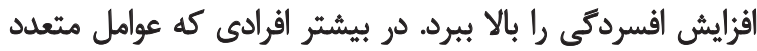

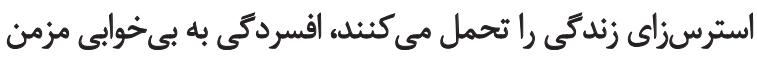

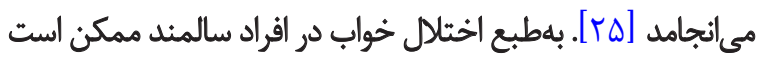

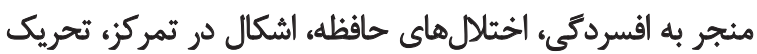

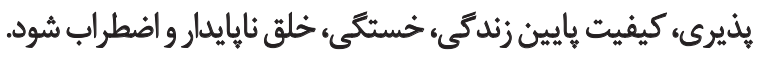

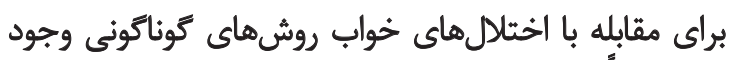

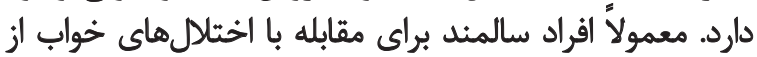

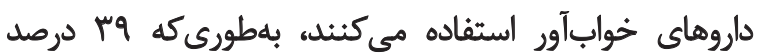

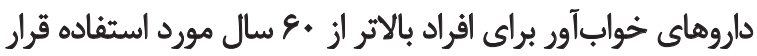

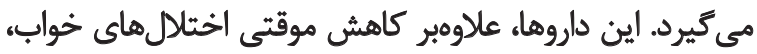

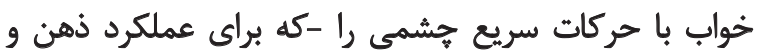

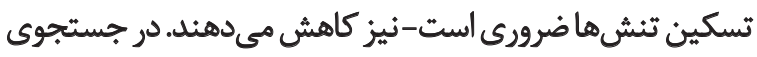

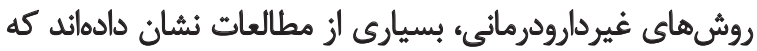

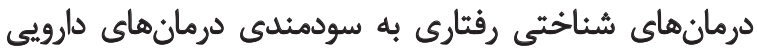

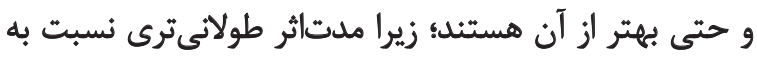

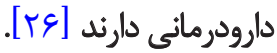

اختلال خواب و انواع آن در خانههاى سالمندان امرى، مثداول

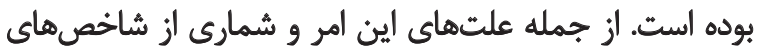

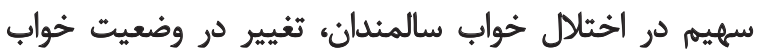

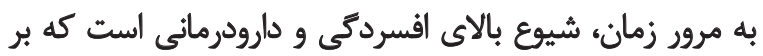

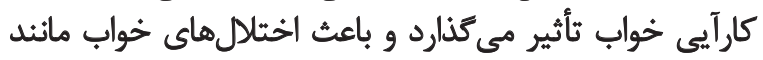

و اختلال در عملكرد روزانه است. درنهايت، بيشترين رابطه با توجه

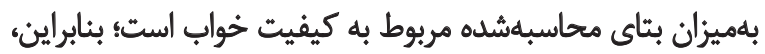

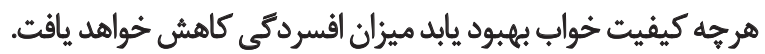

بحث

براساس يافتههاى تحقيق، بين كيفيت خواب و ابعاد آن و واني

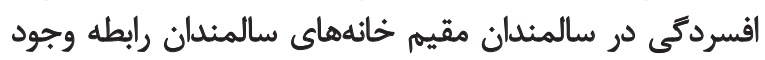

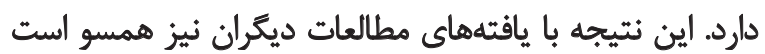

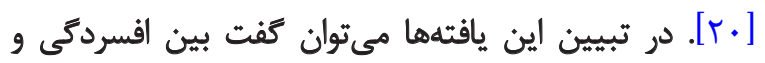

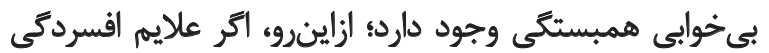

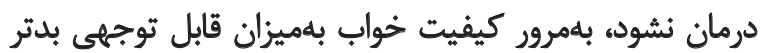

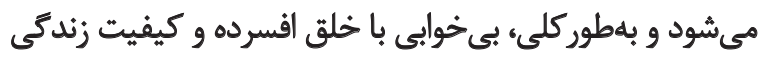

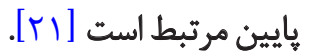

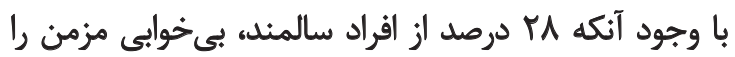

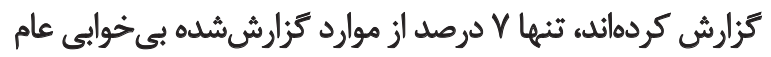

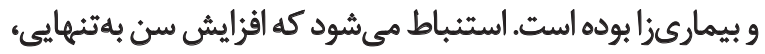

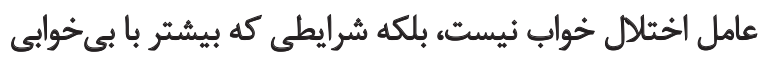

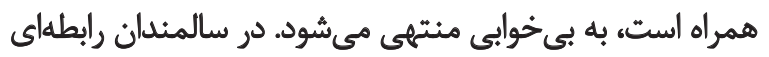

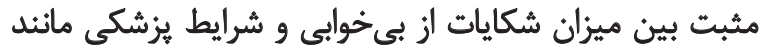

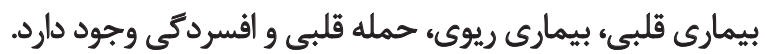

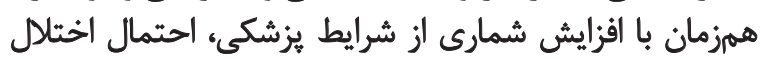

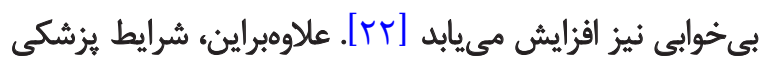

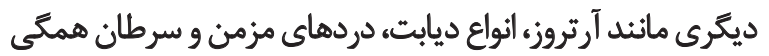

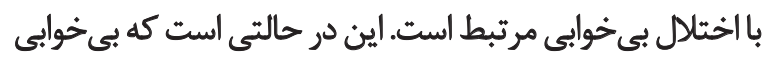

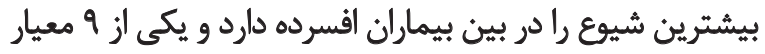
تشخيصى افسردكى بهشمار مي آيد [سب]. براساس مطالعات انجامشده، از افسردكى در سالمندان بهعثوان

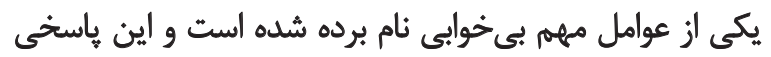

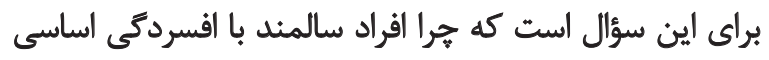




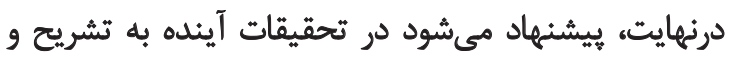

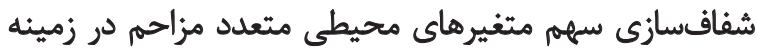

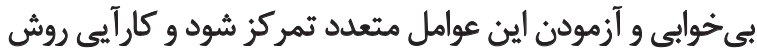

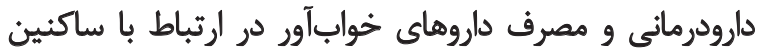

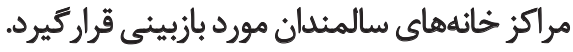

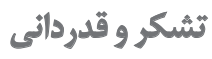

بلينوسيله از مليران سازمان بهزيستي و خانههاي سالمندان

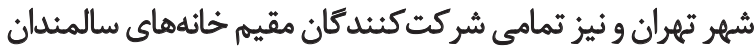

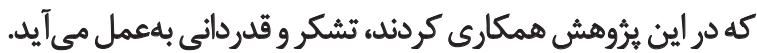

اختلال تنفسى به هنكام خواب مىشود [Yr]. كفتنى است برخى از خانههاي سالمئدان وسايل و امكانات

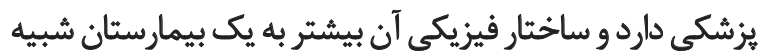

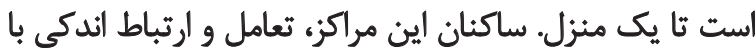

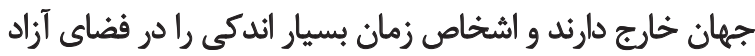

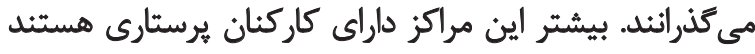

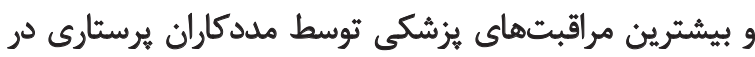

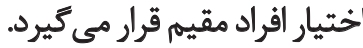

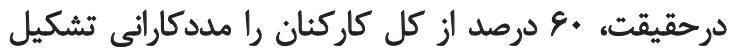

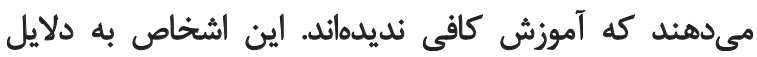

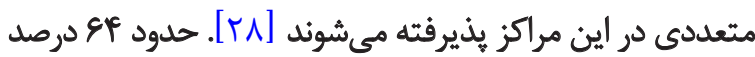

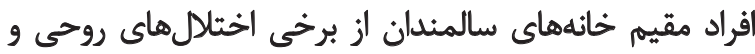

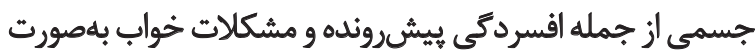

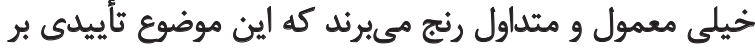

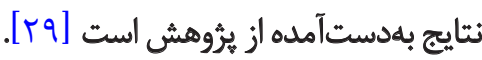

\section{نتيجلئيرى نمهايي}

ضرورت وجود خانههاى سالمندان بهمنظور نتحهدارى سالمندان

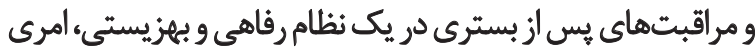

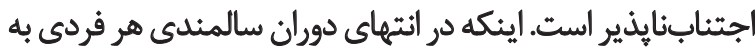

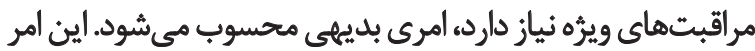

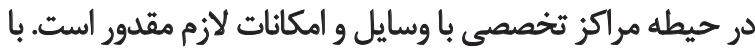

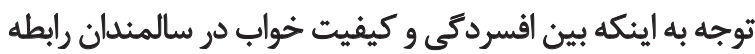

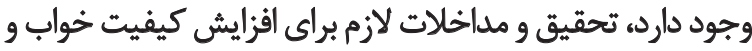

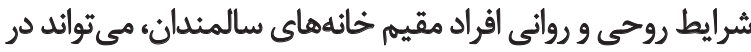
راه بهبود كيفيت خانههاى سالمندان مؤثر باشد.

از محدوديتهاى تحقيق حاضرء مي توان به عدم تعميم نتايج

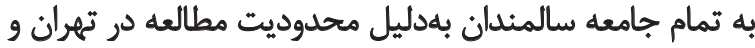

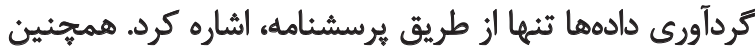

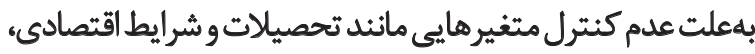

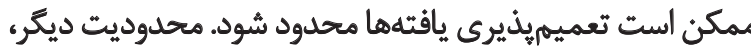

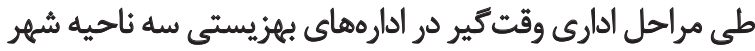

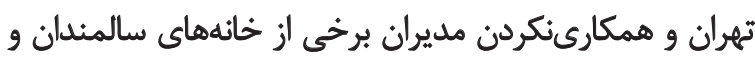
بلمطول|نجاميدن زمان تحقيق است.

ييشنهاد مىشود براي انجام تحقيق در خانههاى سالمندان،

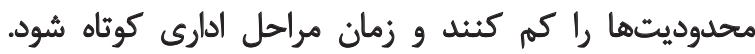

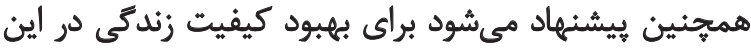

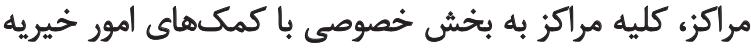

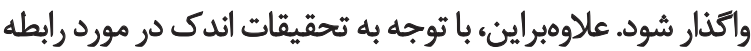

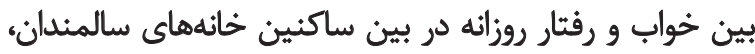
تحقيقات يروهشى بيشترى انجام شود. 


\section{References}

[1] Statistical Center of Iran. General population and housing census. Tehran: Statistical Center of Iran; 2006.

[2] Bogner HR, Shah P, de Vries HF. A cross-sectional study of somatic symptoms and the identification of depression among elderly primary care patients. Primary Care Journal of Clinical Psychiatry. 2009; 11(6):285-91.

[3] Addae-Dapaah K, Juan QS. Life Satisfaction among Elderly Housholds in Public Rental Housing in Singapore. Health. 2014; 6:1057-1076.

[4] Farrell PA. Older people's pain. International Association for the Study of Pain. 2006; 14(3):1-4.

[5] Tannock C, Katona C. Minor depression in the aged. Drugs \& Aging. 1995; 6(4):278-92.

[6] Cotroneo A, Gareri P, Lacava R, Cabodi S. Use of zolpidem in over 75-Year-old Patients with Sleep Disorders and Comorbidities. Archive of Gerontology and Geriatric. 2004; 38(9):93-96.

[7] Ohayon MM, Carskadon MA, Guilleminault C, Vitiello MV. Meta-analysis of Quantitative Sleep Parameters from Childhood to Old Age in Healthy Individuals: Developing Normative Sleep Values Across the Human Lifespan. Sleep. 2004; 22(7):1255-73.

[8] Van Cauter E, Leproult R, Plat L. Age-related changes in slow wave sleep and REM sleep and relationship with growth hormone and cortisol levels in healthy men. Journal of the American Medical Association. 2000; 284(7):861-8.

[9] Ancoli-Israel, S. Insomnia in the Elderly: A Review for the Primary Care Practitioner. Sleep. 2000; 23(1):23-30.

[10] Weyerer S, Dilling H. Prevalence and treatment of insomnia in the community: results from the Upper Bavarian Field Study. Sleep. 1991; 14(5):392-8.

[11] Liu X, Uchiyama M, Kim K, Okawa M, Shibui K, Kudo Y, et al. Sleep loss and daytime sleepiness in the general adult population of Japan. Psychiatry Research. 2000; 93(1):1-11.

[12] Moul DE, Nofzinger EA, Pilkonis PA, Houck PR, Miewald JM, Buysse DJ. Symptom reports in severe chronic insomnia. Sleep. 2002; 25(5):553-63.

[13] Thorpy ML. American Academy of Sleep Medicine. American sleep disorders association, Diagnostic classification steering committee: The international classification of sleep disorders, diagnostic and coding manual. New York: Rochester; 1990.

[14] Komada Y, Nomura T, Kusumi M, Nakashima K, Okajima I, Sasai T, et al. A two-year follow-up study on the symptoms of sleep disturbances/insomnia and their effects on daytime functioning. Sleep Medicine. 2012; 13(9):1115-21.

[15] Babar SI, Enright PL, Boyle P, Foley D, Sharp DS, Petrovitch H, et al. Sleep disturbances and their correlates in elderly Japanese American men residing in Hawaii. Journals of Gerontology Series A: Biological Sciences and Medical Sciences. 2000; 55(7):406-11.

[16] Buysse DJ, Reynolds CF, Kupfer DJ, Thorpy MJ. Clinical diagnoses in 216 insomnia patients using the International Classification of Sleep Disorders (ICSD), DSM-IV and ICD-10 categories: A report from the APA/NIMH DSM-IV Field Trial. Sleep: Journal of Sleep Research \& Sleep Medicine. 1994; 17(7):630-637.
[17] McCullough P. How Well Do You Sleep? Paper presented at: The Kentucky Council of Nurse Practitioners and Midwives; 2001; Lexington, Kentucky, USA.

[18] Buysse DJ, Reynolds CF, Monk TH, Berman SR, Kupfer DJ. The Pittsburgh Sleep Quality Index: A new instrument for psychiatric practice and research. Psychiatry Research. 1989; 28(2):193-213.

[19] Johnson EO, Roth T, Breslau N. The association of insomnia with anxiety disorders and depression: Exploration of the direction of risk. Journal of Psychiatric Research. 2006; 40(8):700-8.

[20] Amini Rastabi Z, Sharifi AK, Refahi J. [Iranian geriatric depression scale: Psychometrics properties (Persian)]. Iranian Journal of Ageing. 2013; 8(2):54-59.

[21] Komada Y, Nomura T, Kusumi M, Nakashima K, Okajima I, Sasai T, et al. Correlations among insomnia symptoms, sleep medication use and depressive symptoms. Psychiatry and Clinical Neurosciences. 2011; 65(1):20-9.

[22] Foley D, Ancoli-Israel S, Britz P, Walsh J. Sleep disturbances and chronic disease in older adults: Results of the 2003 National Sleep Foundation Sleep in America Survey. Journal of Psychosomatic Research. 2004; 56(5):497-502.

[23] American Psychiatric Association. Diagnostic and statistical manual of mental disorders. $4^{\text {th }}$ ed. New York: American Psychiatric Association; 2000.

[24] Roberts RE, Shema SJ, Kaplan GA, Strawbridge WJ. Sleep complaints and depression in an aging cohort: A prospective perspective. American Journal of Psychiatry. 2000; 157(1):81-88.

[25] Cole MG, Dendukuri N. Risk factors for depression among elderly community subjects: Systematic review and meta-analysis. American Journal of Psychiatry. 2003; 160(6):1147-1156.

[26] Alessi CA, Schnelle JF. Approach to sleep disorders in the nursing home setting. Sleep Medicine Reviews. 2000; 4(1):45-56.

[27] Hing E, Sekscenski E, Strahan G. The National Nursing Home Survey: 1985 Summary for the United States. Vital and Health Statistics. Series 13, Data from the National Center for Health Statistics. 1989; 97(1):89-1758.

[28] Ancoli-Israel S, Parker L, Sinaee R, Fell RL, Kripke DF. Sleep fragmentation in patients from a nursing home. Journal of Gerontology. 1989; 44(1):18-21.

[29] Léger D, Scheuermaier K, Philip P, Paillard M, Guilleminault C. SF-36: Evaluation of quality of life in severe and mild insomniacs compared with good sleepers. Psychosomatic medicine. 2001; 63(1):49-55. 